\title{
Molecular rotation in 3 dimensions at an air/ water interface using femtosecond time resolved sum frequency generation
}

Cite as: J. Chem. Phys. 150, 094709 (2019); https://doi.org/10.1063/1.5080228

Submitted: 06 November 2018 . Accepted: 15 February 2019 . Published Online: 07 March 2019

Yi Rao (D), Yuqin Qian, Gang-Hua Deng, Ashlie Kinross, Nicholas J. Turro, and Kenneth B. Eisenthal (D)

\section{ARTICLES YOU MAY BE INTERESTED IN}

The dielectric constant: Reconciling simulation and experiment

The Journal of Chemical Physics 150, 084108 (2019); https://doi.org/10.1063/1.5080927

Development of ultrafast broadband electronic sum frequency generation for charge dynamics at surfaces and interfaces

The Journal of Chemical Physics 150, 024708 (2019); https://doi.org/10.1063/1.5063458

A rate-doubled 10-GHz fiducial comb generator for precision optical timing calibration

Review of Scientific Instruments 90, 035103 (2019); https://doi.org/10.1063/1.5086159

Where in the world is AIP Publishing?

Find out where we are exhibiting next 


\title{
Molecular rotation in 3 dimensions at an air/water interface using femtosecond time resolved sum frequency generation
}

\author{
Cite as: J. Chem. Phys. 150, 094709 (2019); doi: 10.1063/1.5080228 \\ Submitted: 6 November 2018 • Accepted: 15 February 2019 • \\ Published Online: 7 March 2019
}

Yi Rao, ${ }^{1, a)}$ (D) Yuqin Qian, ${ }^{1}$ Gang-Hua Deng, ${ }^{1}$ Ashlie Kinross, ${ }^{1}$ Nicholas J. Turro, ${ }^{2}$ and Kenneth B. Eisenthal ${ }^{2}$

\author{
AFFILIATIONS \\ ${ }^{1}$ Department of Chemistry and Biochemistry, Utah State University, Logan, Utah 84322, USA \\ ${ }^{2}$ Department of Chemistry, Columbia University, New York, New York 10025, USA
}

Note: This article is part of the Special Topic "Nonlinear Spectroscopy and Interfacial Structure and Dynamics" in J. Chem. Phys.

a) Authors to whom correspondence should be addressed: yi.rao@usu.edu and kbet@columbia.edu.

\begin{abstract}
This paper presents the first study of the rotations of rigid molecules in 3 dimensions at the air/water interface, using the femtosecond time resolved sum frequency generation (SFG) technique. For the purpose of this research, the aromatic dye molecule C153 was chosen as an example of a molecule having two functional groups that are SFG active, one being the hydrophilic $-\mathrm{C}=\mathrm{O}$ group and the other the hydrophobic $-\mathrm{CF}_{3}$ group. From polarized SFG measurements, the orientations of the two chromophores with respect to the surface normal were obtained. On combining these results with the known relative orientation of the two chromophores in the molecule yields the absolute orientation of $\mathrm{C} 153$ at the air/water interface. It was found that the $-\mathrm{CF}_{3}$ axis projected towards the bulk air at an angle of $59^{\circ}$ with respect to the interface normal and the $-\mathrm{C}=\mathrm{O}$ group projected towards the bulk water at an angle of $144^{\circ}$. In order to observe the rotational motions of $\mathrm{C} 153$ at the air/water interface, the approach was used to perturb the ground electronic state equilibrium orientational distribution using a polarized resonant pump pulse, which preferentially excites ground state molecules that have their electronic $S_{0} \rightarrow S_{1}$ transition moment aligned closely to the electric field of the incident pump pulse. As a consequence of the photoselection perturbation, the orientational distribution of the remaining ground state molecules was not the equilibrium distribution. Similarly, the orientational distribution of the excited state molecules that were created by the polarized pump pulse was not in their final equilibrium orientational distribution. The rotational motions of the interfacial molecules towards equilibrium were obtained from time dependent measurements of the intensities of the SFG signal generated by the simultaneous incidence at the air/water interface of a visible probe pulse plus an IR probe pulse. In this way, the recovery times to achieve the orientational equilibrium of the two chromophores including the orientation of the normal of the $\mathrm{C} 153$ plane with respect to the interface were obtained. The photo-selection process shifts the average orientation angle of the hydrophilic $-\mathrm{C}=\mathrm{O}$ group by an increase of $4^{\circ} \pm 0.6^{\circ}$ with a rotational recovery time constant of $130 \pm 20 \mathrm{ps}$, which is the time to return to an orientational equilibrium distribution. The hydrophobic $-\mathrm{CF}_{3}$ group undergoes a shift that increases its angle by $8^{\circ} \pm 1.5^{\circ}$ with a rotational recovery time constant of $210 \pm 38$ ps. We find that the orientational change of the molecular normal is $4^{\circ} \pm 0.5^{\circ}$ and has a rotational recovery time constant of $125 \pm 26$ ps. The interface-specific time-dependent polarized measurements allowed us to monitor the orientational motions of molecules at interfaces, both in 3 dimensions and in real time.
\end{abstract}

Published under license by AIP Publishing. https://doi.org/1 0.1063/1.5080228

\section{INTRODUCTION}

Interfaces are the molecularly thin regions of matter which separate distinct physical and/or chemical bulk regions of matter. Intrinsic anisotropic geometry of interfaces is the origin of their unique chemical, physical, and biological properties. Water interfaces are predominant on our planet, of which the air/water interface is the most important in covering more than $2 / 3$ of the Earth's surface. Of note are the mineral oxide/water interfaces as that is an important location where soil chemistry chiefly takes place. Of 
special significance are biological cell membrane/water interfaces as they are central to life processes.

Interfaces not only have different properties from the regions that bound them but also serve as the gate keeper that controls the passage of all energy, radiation, and chemical species between the adjacent phases. The lower symmetry of the interface plays a key role in determining the orientational structure, chemical composition, chemical and physical equilibria, and other time independent properties as well as time dependent molecular motions, chemical transformations, energy and charge transfer, and others.

Second Harmonic Generation (SHG) is a second order optical process that depends primarily on the electronic properties of the interfacial molecules, while Sum Frequency Generation (SFG) is another second order optical process which is sensitive to molecular vibrations when one incident beam is tuned in the infrared region that corresponds to an interfacial SFG allowed vibration. Both processes can be employed to specifically investigate interfaces that are accessible to light, including buried interfaces. $^{1-4}$ Static SHG and SFG measurements provide valuable information about the populations of interfacial species, molecular orientational ordering, acid-base equilibrium, etc., occurring at an interface. ${ }^{5-24}$ Time-resolved SHG has also been used to study solvation dynamics, rotational dynamics, electron transfer, population recovery, two-dimensional energy transfer, and other ultra-fast processes. ${ }^{25-30}$ SFG is a powerful method to investigate structural dynamics, vibrational energy relaxation, thermal transport, and other interfacial processes. ${ }^{19,31-37}$ In recent work, we have used visible-pump-time-resolved vibrational SFG experiments to study rotational dynamics of a single chemical group of a molecule at the air/water interface using the visible-pump-SFG probe technique. $^{32}$ In the work reported here, we exploit this technique to observe the rotational dynamics of a molecule in 3 dimensions by measuring the rotational dynamics of two chromophores of a selected molecule which are not collinearly oriented on the molecule.

Molecules assume orientations that lower the free energy in the interfacial region. At the air/water interface, this orientation generally will align a polar non-hydrogen bonding adsorbate with its more polar part projecting into the water phase and the less polar part towards the air. After excitation, molecular motions occur returning the adsorbates back to the lower free energy orientation. The orientations and rotational motions of interfacial molecules can be utilized as sensitive probes of the unique asymmetric environment that interfacial chemical species experience. For hydrogen bonding adsorbates such as phenol, we have found from absolute phase measurements that the hydroxyl group points down (towards the water), which is also the direction of the permanent dipole moment of phenol. Unlike phenol both nitrophenol and bromophenol have their permanent dipole moments in the opposite direction to that of phenol. The question is then "Is it the permanent dipole moment or is it the hydrogen bonding hydroxyl group that determines the up towards the air vs down towards the water orientation?" Phase measurements in our lab showed that phenol, bromophenol, and nitrophenol all have their hydroxyl group pointing towards the bulk water. ${ }^{38}$ This displays the hydrogen bonding dominance. It is the hydrogen bonding of the hydroxyl group with water rather than the dipole moment direction that determines the up-down alignment of these molecules at the air/water interface.

The in-plane orientational distribution of adsorbates at the air/water interface is, on average, isotropic because the in-plane intermolecular forces are, on average, isotropic in the interfacial plane. The intermolecular forces that are out-of-plane with respect to the interface, i.e., the air above and water below, are anisotropic. This anisotropy is responsible for the adsorbate orientation, given by the polar angle $\theta$. Just as the equilibrium orientational distribution in $\theta$ is restricted by the asymmetry of the interfacial potential, the translational and rotational motions of interfacial molecules are restricted by the asymmetric potential of the interface. Unlike the bulk water, in which rotational motions occur in all directions with equal probability, the dynamics of rotation in the interfacial plane differ from rotations out of the interfacial plane. In earlier SHG studies of orientational relaxation, the rotation dynamics of a single symmetry axis about the interfacial normal ${ }^{29,30,39-41}$ was obtained. In the work reported here, the objective was to measure the interfacial rotation dynamics of the whole molecule, i.e., rotation of the molecule in three dimensions. Employing a procedure used in earlier work, ${ }^{42}$ we obtained the absolute orientation of the interfacial molecule. This was accomplished by measuring the orientations with respect to the surface normal of two SFG active vibrational chromophores that are not collinear in a rigid molecule. Furthermore, it was assumed that the up vs. down alignment of the molecule in the interface is known. It was assumed that the orientational distribution is given by a delta function. This will be briefly discussed at a later point.

Experiments using time-resolved sum frequency measurements were used to obtain the absolute orientation of two chromophores as the molecules rotate toward their equilibrium orientations. Time-resolved polarized SFG experiments used a pump pulse to photoselect an orientational subset of the equilibrium distribution of ground state molecules, which thereby generate a non-equilibrium orientational distribution in both the ground state molecules that were not excited and the excited state molecules produced by the pump pulse. ${ }^{27,28,30,32,39,40}$ Depending on the polarization of the pump pulse, the change in the SFG intensity with time can depend on both in-plane and out-of-plane rotations. It has been shown that the out-of-plane rotations can be separated from the in-plane rotations by using a circularly polarized laser pump pulse that is incident normal to the interfacial plane. ${ }^{40}$ At a resonant pump frequency, the interfacial molecules were excited to their lowest excited singlet state. The ground state molecules, prior to the pump pulse, were isotropically distributed in the interfacial plane because of the inherent in-plane isotropy of forces at the air/water interface. The circularly polarized pump light has the same intensity in all directions parallel to the interface plane. Both the remaining ground and excited state molecules were at all times isotropically distributed in the interface plane. The in-plane distribution of molecules, therefore, has no time-dependent change because these molecules are always isotropic in the plane of the interface. However, because the pump light preferentially excites molecules whose transition moments are parallel to the polarization of the pump electric field, the out-of-plane orientational distribution of the ground state molecules that were and were not excited is not in their equilibrium orientational distributions. It is the evolution of the out-ofplane orientational distribution from nonequilibrium to equilibrium that is achieved by rotational motions of the interfacial molecules 
that we observed. To study the in-plane rotational dynamics, the first step is to break the initial isotropic symmetry of the in-plane orientational distribution. This was achieved by using a linearly polarized pump light, e.g., along the in-plane $\mathrm{X}$-axis, which photoinduces an in-plane orientational anisotropy (fewer unexcited ground state molecules oriented along the X-axis). In the work reported here, we will mainly focus on the out-of-plane motion of molecules at the air/water interface.

\section{THEORETICAL CONSIDERATIONS}

\section{A. Static SFG and interfacial order parameter $D(-\infty)$ at equilibrium}

The SFG intensity of any of the polarization combinations at equilibrium, $I^{S F G}$, is related to the surface second order susceptibility $\chi^{(2)}$. The susceptibility is the sum over all macroscopic sum frequency susceptibility terms separated into a non-resonant part $\chi_{N R}^{(2)}$ and a resonant part $\chi_{R}^{(2)}$. Thus, one is able to express the SFG intensity as the following generalized expression: ${ }^{3}$

$$
I^{S F G} \propto\left|\chi_{R}^{(2)}+\chi_{N R}^{(2)}\right|^{2}
$$

In the case of SFG experiments, a tunable or broadband IR beam, which is resonant with the chemical groups of interest, enhances the susceptibility as expressed in the following: ${ }^{3}$

$$
\begin{aligned}
& \chi^{(2)}=\chi_{N R}^{(2)}+\sum_{q} \chi_{R, q}^{(2)}, \\
& \chi_{R, q}^{(2)}=\frac{A_{q}}{\omega-\omega_{q}+i \Gamma_{q}},
\end{aligned}
$$

where $\omega_{q}$ denotes the resonant frequency of the $q$ th vibrational normal mode, and $\Gamma_{\mathrm{q}}$ is the dephasing rate of the vibrational state, $A_{q}$ contains the product of the Raman and infrared matrix elements of the $q$ th normal mode. The resonant second order susceptibility of interfaces, $\chi_{R}^{(2)}$, is related to molecular hyperpolarizability $\alpha_{i j k}^{(2)}$ by spatial transformation from a molecular coordinate frame to the laboratory coordinate frame (Fig. 1) 43

$$
\begin{aligned}
\chi_{I J K}^{(2)} & =N \sum_{i j k}\left\langle R_{I i} R_{J j} R_{K k}\right\rangle \alpha_{i j k}^{(2)}, \\
\alpha_{q, i j k}^{(2)} & =\frac{a_{q, i j k}}{\omega-\omega_{q}+i \Gamma_{q}},
\end{aligned}
$$

where $R_{I i}, R_{J j}$, and $R_{K k}$ are the direction cosine matrices that transform the laboratory coordinate frame $(I, J, K=X, Y, Z)$ into the

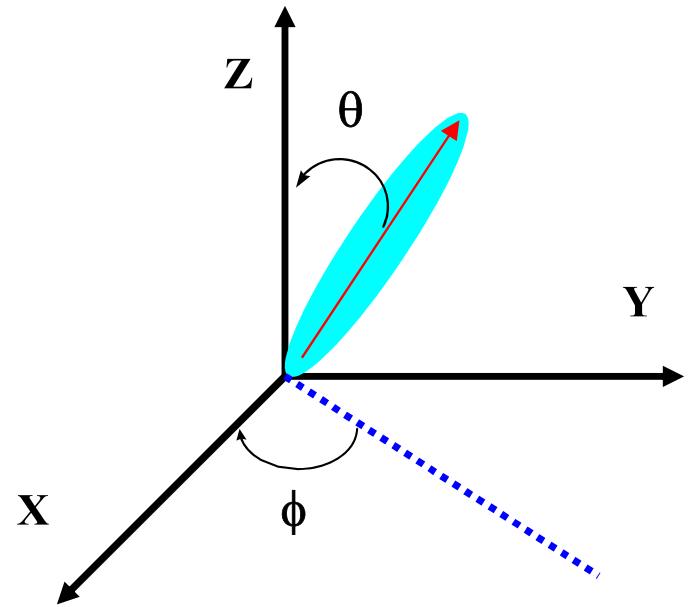

FIG. 1. A polar orientation of a dipole in the laboratory coordinate frame.

molecular coordinate frame $(i, j, k=x, y, z), N$ is the surface density of ground-state molecules at equilibrium, and \langle\rangle denotes the ensemble average of the orientational distribution $\rho_{g}(\theta, t<0)$. In SHG, it is often assumed, with some justification, that the SHG hyperpolarizability $\alpha^{(2)}$ can be treated as uniaxial with only one element $\alpha_{z z z}^{(2)}$ when the SHG wavelength is strongly resonant with a transition along the molecular axis $z$. However, in the case of the vibrationally resonant SFG, the hyperpolarizability $\alpha^{(2)}$ of a vibrational dipole cannot be simply considered as a single element, depending upon the symmetry of the chemical groups of interest. This arises because the electronic polarizabilities of chemical bonds, which are a measure of the response of the electrons to an external field, are usually anisotropic. Of special interest are three kinds of symmetries including $\mathrm{C}_{\infty \mathrm{v}}, \mathrm{C}_{2 \mathrm{v}}$, and $\mathrm{C}_{3 \mathrm{v}}$, which represent a majority of functional chemical groups. For example, there are 11 nonzero microscopic hyperpolarizability elements of the $\mathrm{C}_{3 \mathrm{v}}$ symmetry group such as the $-\mathrm{CF}_{3}$ group, three corresponding to symmetric stretching modes and eight to asymmetric stretching modes. ${ }^{17,44,45}$ For $\mathrm{C}_{2 \mathrm{v}}$ symmetry groups, such as the $-\mathrm{CH}_{2}$ group, there are 7 non-vanishing molecular hyperpolarizability tensor elements, three contributing to symmetric stretching modes, and four to asymmetric stretching modes. The nonzero hyperpolarizability terms for a $\mathrm{C}_{\infty \mathrm{v}}$ symmetry group such as $-\mathrm{C}=\mathrm{O},-\mathrm{CN},-\mathrm{CH}$, etc., are all symmetric, with $\alpha_{x x z}^{(2)}=\alpha_{y y z}^{(2)}=r^{*} \alpha_{z z z}^{(2)}$, where $r=\alpha_{x x z}^{(2)} / \alpha_{z z z}^{(2)} \cdot 44-46$

At liquid surfaces, only the seven elements $\chi_{X Z X}^{(2)}=\chi_{Y Z Y}^{(2)}, \chi_{Z X X}^{(2)}$ $=\chi_{Z Y Y}^{(2)}, \chi_{X X Z}^{(2)}=\chi_{Y Y Z}^{(2)}$, and $\chi_{Z Z Z}^{(2)}$ are nonzero (the $X$ and $Y$ axes are equivalent for an isotropic surface). ${ }^{2}$ Thus, before photoexcitation, the second order susceptibilities of their symmetric stretching modes are expressed as follows: ${ }^{17,43}$

$$
\begin{aligned}
& \chi_{X X Z}^{(2)}(-\infty)=\chi_{Y Y Z}^{(2)}(-\infty)=\frac{1}{2} N \alpha_{z z z}^{(2)}\left[(1+r)\langle\cos \theta(-\infty)\rangle-(1-r)\left\langle\cos ^{3} \theta(-\infty)\right\rangle\right], \\
& \chi_{X Z X}^{(2)}(-\infty)=\chi_{Y Z Y}^{(2)}(-\infty)=\chi_{Z Y Y}^{(2)}(-\infty)=\chi_{Z X X}^{(2)}(-\infty)=\frac{1}{2} N \alpha_{z z Z}^{(2)}(1-r)\left[\langle\cos \theta(-\infty)\rangle-\left\langle\cos ^{3} \theta(-\infty)\right\rangle\right], \\
& \chi_{Z Z Z}^{(2)}(-\infty)=N \alpha_{Z Z Z}^{(2)}\left[r\langle\cos \theta(-\infty)\rangle+(1-r)\left\langle\cos ^{3} \theta(-\infty)\right\rangle\right] .
\end{aligned}
$$


The four polarization combinations measured in each SFG experiment were SSP, $P P P, S P S$, and PSS, where the first letter denotes the polarization of the sum frequency light, the second one denotes the polarization of the $400 \mathrm{~nm}$ light, and the last one denotes the polarization of the IR light. These polarizations are connected with the laboratory coordinates by nonlinear Fresnel coefficients and appropriate projections. The four effective susceptibilities for SFG experiments (SSP, SPS, PSS, and PPP) are related to the above seven second order susceptibilities by local field factors $L_{i i}$ as expressed, ${ }^{17,47}$

$$
\begin{aligned}
\chi_{S S P}^{(2)}(-\infty)= & L_{X X} L_{X X} L_{Z Z} \sin \beta_{3} \chi_{X X Z}^{(2)}(-\infty), \\
\chi_{S P S}^{(2)}(-\infty)= & L_{X X} L_{Z Z} L_{X X} \sin \beta_{2} \chi_{X Z X}^{(2)}(-\infty), \\
\chi_{P S S}^{(2)}(-\infty)= & L_{Z Z} L_{X X} L_{X X} \sin \beta_{1} \chi_{Z X X}^{(2)}(-\infty), \\
\chi_{P P P}^{(2)}(-\infty)= & L_{Z Z} L_{Z Z} L_{Z Z} \sin \beta_{1} \sin \beta_{2} \sin \beta_{3} \chi_{Z Z Z}^{(2)}(-\infty)+L_{Z Z} L_{Y Y} L_{Y Y} \sin \beta_{1} \cos \beta_{2} \cos \beta_{3} \chi_{Z Y Y}^{(2)}(-\infty) \\
& -L_{Y Y} L_{Z Z} L_{Y Y} \cos \beta_{1} \sin \beta_{2} \cos \beta_{3} \chi_{Y Z Y}^{(2)}(-\infty)-L_{Y Y} L_{Y Y} L_{Z Z} \cos \beta_{1} \cos \beta_{2} \sin \beta_{3} \chi_{Y Y Z}^{(2)}(-\infty) .
\end{aligned}
$$

By substituting Eq. (4) into Eq. (5) and then into Eq. (1), the surface susceptibility of any polarization combinations before photoexcitation, $\chi_{\delta \eta \xi}^{(2)}(-\infty)$, can be simply formulated as the following generalized expression: ${ }^{27,42,48}$

$$
\chi_{\delta \eta \xi}^{(2)}(-\infty)=N A_{\delta \eta \xi}\left(\langle\cos \theta(-\infty)\rangle-c_{\delta \eta \xi}\left\langle\cos ^{3} \theta(-\infty)\right\rangle\right) \alpha_{z z z}^{(2)},
$$

where the parameters $A$ and $c$ are functions of the incident and outgoing angles and the polarization angles, and the dielectric constants of both the fundamental and second harmonic light frequencies in the bulk media and in the molecular layer.

According to Eq. (6), one can measure an interfacial order parameter $D(\mathrm{t})$. At equilibrium (before photo-excitation), its value can be obtained from the ratio of any two of the four polarization SFG intensities or from null-angle measurements, yielding at $t=-\infty$,

$$
D(-\infty)=\frac{\langle\cos \theta(-\infty)\rangle}{\left\langle\cos ^{3} \theta(-\infty)\right\rangle}
$$

The utility of the order parameter $D$ for a delta function orientational distribution is that the measured value of $D$ yields the orientational angle $\theta$.

\section{B. Transient SFG and time-dependent interfacial order parameter $D(t)$}

The time evolution of the various relaxation processes following photoexcitation includes ultrafast solvation dynamics, orientational dynamics, and population recovery, all of which are well separated in time in our experiments. The solvation dynamics, which for Coumarin 314 and Coumarin 153 are a few picoseconds in duration at the air/water interface, have been discussed previously. ${ }^{31,49,50}$ The time evolution of the orientational dynamics is often described by the conditional probability $G\left(\theta_{t}, t ; \theta_{0}, 0\right)$ for the molecules that have the orientation $\theta_{t}$ at the time $t$ when it had the orientation $\theta_{0}$ at the initial time $t=0$. Thus, the time evolution of the orientational population for the non-equilibrium ground state is given by, $G\left(\theta_{t}, t ; \theta, 0\right) \rho_{g}(\theta,-\infty) N_{g}(t-\infty)(1-$ $\left.\sigma|\mu|^{2}|E|^{2} \sin ^{2} \theta_{0}\right),{ }^{30}$ where $\sigma$ is a collection of constants associated with photo-excitation, $\mu$ is the transition dipole moment, and $E$ is the electric field of the normal incident circularly polarized pump pulse. $^{30}$

The susceptibility element $\chi_{X Z X}^{(2)}$ for the perturbed ground state, which corresponds to the SPS polarization combination, is taken as an example. For the perturbed ground state, we can write the timedependent susceptibilities as follows: ${ }^{27}$

$$
\begin{aligned}
\chi_{g, X Z X}^{(2)}(t) & =A_{X Z X} \alpha_{g, \text { zZZ }}^{(2)} \int d \theta_{t} \sin \theta_{t} \int d \theta_{0} \sin \theta_{0}\left(\cos \theta_{t}-c_{X Z X} \cos ^{3} \theta_{t}\right) N_{g}(\theta, t>0) \\
& =A_{X Z X} N_{g}(-\infty) \alpha_{g, \mathrm{zZZ}}^{(2)} \int d \theta_{t} \sin \theta_{t} \int d \theta_{0} \sin \theta_{0}\left(\cos \theta_{t}-c_{X Z X} \cos ^{3} \theta_{t}\right) G\left(\theta_{t}, \mathrm{t} ; \theta_{0}, 0\right) \rho_{g}(\theta,-\infty)\left(1-\sigma|\mu|^{2}|E|^{2} \sin ^{2} \theta_{0}\right) \\
& =A_{X Z X} \chi_{g, X Z X}^{(2)}(-\infty)-A_{X Z X} N_{g}(-\infty) \alpha_{g, \mathrm{zzZ}}^{(2)} \int d \theta_{t} \sin \theta_{t} \int d \theta_{0} \sin \theta_{0}\left(\cos \theta_{t}-c_{X Z X} \cos ^{3} \theta_{t}\right) G\left(\theta_{t}, \mathrm{t} ; \theta_{0}, 0\right) \rho_{g}(\theta,-\infty) \sigma|\mu|^{2}|E|^{2} \sin ^{2} \theta \\
& =\chi_{g, X Z X}^{(2)}(-\infty)-A_{X Z X} N_{g}(-\infty) \sigma|\mu|^{2}|E|^{2} \alpha_{g, \text { zzz }}^{(2)} \int d \theta_{t} \sin \theta_{t} \int d \theta_{0} \sin \theta_{0}\left(\cos \theta_{t}-c_{X Z X} \cos ^{3} \theta_{t}\right) G\left(\theta_{t}, \mathrm{t} ; \theta_{0}, 0\right) \rho_{g}(\theta,-\infty) \sin ^{2} \theta_{0} \\
& =\chi_{g, X Z X}^{(2)}(-\infty)-A_{X Z X} N_{g}(-\infty) \sigma|\mu|^{2}|E|^{2} \alpha_{g, \mathrm{zzZ}}^{(2)}\left(\langle\cos \theta(t)\rangle-c_{X Z X}\left\langle\cos ^{3} \theta(t)\right\rangle\right),
\end{aligned}
$$


where

$$
\begin{aligned}
& \langle\cos \theta(t)\rangle=\int d \theta_{t} \sin \theta_{t} \int d \theta_{0} \sin \theta_{0} \cos \theta_{t} G\left(\theta_{t}, \mathrm{t} ; \theta_{0}, 0\right) \rho_{g}(\theta,-\infty) \sin ^{2} \theta_{0}, \\
& \left\langle\cos ^{3} \theta(t)\right\rangle=\int d \theta_{t} \sin \theta_{t} \int d \theta_{0} \sin \theta_{0} \cos ^{3} \theta_{t} G\left(\theta_{t}, \mathrm{t} ; \theta_{0}, 0\right) \rho_{g}(\theta,-\infty) \sin ^{2} \theta_{0} .
\end{aligned}
$$

The concrete formula for $A_{\mathrm{xzx}}$ and $C_{\mathrm{xzx}}$ as well as $A$ and $C$ for other polarization combinations is found in the previous work. ${ }^{27,48,51-53}$ In this work, the resultant SFG wavelengths for the $-\mathrm{C}=\mathrm{O}$ and $-\mathrm{CF}_{3}$ group of coumarin 153 fall at ca. $375 \mathrm{~nm}$ and $380 \mathrm{~nm}$, respectively. The fundamental light of $400 \mathrm{~nm}$ picosecond light and the SFG lights are close to the maximum absorption peak of coumarin 153 of interest. As such, the ground state SFG experiments here are considered as near doubly resonant SFG. ${ }^{54}$

Likewise, the time evolution of the orientational population for the non-equilibrium excited state is given by $G\left(\theta_{t}, \mathrm{t} ; \theta_{0}, 0\right) \rho_{g}(\theta,-\infty)$ $N_{g}(-\infty) \sigma|\mu|^{2}|E|^{2} \sin ^{2} \theta_{0}$. Thus, the time-dependent susceptibility for the excited state is expressed as

$$
\begin{aligned}
\chi_{e, X Z X}^{(2)}(t) & =A_{X Z X} \alpha_{e, \mathrm{zzZ}}^{(2)} \int d \theta_{t} \sin \theta_{t} \int d \theta_{0} \sin \theta_{0}\left(\cos \theta_{t}-c_{X Z X} \cos ^{3} \theta_{t}\right) N_{e}(\theta, t>0) \\
& =A_{X Z X} \alpha_{e, \mathrm{zzZ}}^{(2)} \int d \theta_{t} \sin \theta_{t} \int d \theta_{0} \sin \theta_{0}\left(\cos \theta_{t}-c_{X Z X} \cos ^{3} \theta_{t}\right) G\left(\theta_{t}, \mathrm{t} ; \theta_{0}, 0\right) \rho_{g}(\theta,-\infty) N_{g}(-\infty) \sigma|\mu|^{2}|E|^{2} \sin ^{2} \theta_{0} \\
& =A_{X Z X} N_{g}(-\infty) \sigma|\mu|^{2}|E|^{2} \alpha_{e, \mathrm{zzZ}}^{(2)} \int d \theta_{t} \sin \theta_{t} \int d \theta_{0} \sin \theta_{0}\left(\cos \theta_{t}-c_{X Z X} \cos ^{3} \theta_{t}\right) G\left(\theta_{t}, \mathrm{t} ; \theta_{0}, 0\right) \rho_{g}(\theta,-\infty) \sin ^{2} \theta_{0} \\
& =A_{X Z X} N_{g}(-\infty) \sigma|\mu|^{2}|E|^{2} \alpha_{e, \mathrm{zzz}}^{(2)}\left(\left\langle\cos \theta_{t}\right\rangle-c_{X Z X}\left\langle\cos ^{3} \theta_{t}\right\rangle\right)
\end{aligned}
$$

After excitation, the total susceptibility is ${ }^{30,32}$

$$
\chi_{\text {total, } X Z X}^{(2)}(t)=\chi_{g, X Z X}^{(2)}(t)+\chi_{e, X Z X}^{(2)}(t) .
$$

Although both the ground state and the excited state could contribute to the time-dependent responses, the transient signal from the ground state dominates upon photoexcitation. For transient absorption spectroscopy (or pump-probe) in bulk, ground state bleaching (GSB) and ground state recovery (GSR), excited state absorption (ESA) and stimulated emission (SE) are expected to be observed after photoexcitation. GSB/GSR and SE show negative spectral features in transient absorption spectra, whereas ESA exhibits positive response in the absorption. For the transient SFG experiments, we anticipated to observe the similar behaviors of the photoexcited molecules at interfaces to those in bulk. In particular, a red shift in SFG spectra of excited states is anticipated upon photoexcitation. However, we did not find any spectral shift but only decrease in SFG signals at different time delays upon photoexcitation of coumarin 153 at the air/water interface. There were some possibilities that we could not observe any spectral shift in the excited state of coumarin 153. First of all, the hyperpolarizabilities for the $-\mathrm{C}=\mathrm{O}$ and $-\mathrm{CF}_{3}$ groups in coumarin 153 for excited states might be too weak to be observed under our experimental conditions. Although our experimental detection limit was on the order of $1 \%$ for the change in SFG signal, it might not be sensitive enough to detect weak signals from the excited states. Second, a spectral shift in excited states for the two groups could be too small to be seen in our experimental case. Third, signal-to-noise ratio improvements in collection and sampling methods, or laser systems, are required in the future to observe spectral shifts in excited states. Based on our experimental observations and those above-mentioned possibilities, we suggested that the time-dependent SFG signal is primarily from the recovery of the functional groups of coumarin 153 in the ground state.

Thus, the transient SFG intensity of the SPS polarization combination with a circularly polarized pump pulse propagating along the normal to the surface can be expressed by the following:

$$
\begin{aligned}
I_{S F G}^{X Z X}(t) & \left.\propto\left|\chi_{g, X Z X}^{(2)}(-\infty)-A_{X Z X} N_{g}(-\infty) \sigma\right| \mu\right|^{2}|E|^{2} \alpha_{g, \mathrm{zzZ}}^{(2)}\left[\left.\left(\langle\cos \theta(t)\rangle-c_{X Z X}\left\langle\cos ^{3} \theta(t)\right\rangle\right)\right|^{2}\right. \\
& \approx I_{S F G}^{X Z X}(-\infty)-2 A_{X Z X} \chi_{g, X Z X}^{(2)}(-\infty) \alpha_{g, \mathrm{zzZ}}^{(2)} N_{g}(-\infty) \sigma|\mu|^{2}|E|^{2} \alpha_{g, \mathrm{zzZ}}^{(2)}\left(\langle\cos \theta(t)\rangle-c_{X Z X}\left\langle\cos ^{3} \theta(t)\right\rangle\right) .
\end{aligned}
$$

The change of time-dependent SFG with respect to that at equilibrium is given by the following expression for the SSP intensity measurements: 


$$
\begin{aligned}
\frac{\Delta I_{S S P}^{(2)}(t)}{I_{S S P}^{(2)}(-\infty)} & =\frac{I_{S S P}^{(2)}(t)-I_{S S P}^{(2)}(-\infty)}{I_{S S P}^{(2)}(-\infty)} \\
& \approx-\frac{2 \sigma|\mu|^{2}|E|^{2}}{N_{g}(-\infty)} \frac{\langle\cos \theta(t)\rangle-c_{X X Z}\left\langle\cos ^{3} \theta(t)\right\rangle}{\langle\cos \theta(-\infty)\rangle-c_{X X Z}\left\langle\cos ^{3} \theta(-\infty)\right\rangle}
\end{aligned}
$$

Experimentally, the quantities of interest are the changes in the intensities of $S S P$ and $S P S$ at different delay times $t$, namely, $\frac{\Delta I_{S S P}^{(2)}(t)}{I_{S S P}^{(2)}(-\infty)}$ and $\frac{\Delta I_{S P S}^{(2)}(t)}{I_{S P S}^{(2)}(-\infty)}$. By dividing the SSP and SPS terms by their value at $\mathrm{t}=0$, one obtains the following:

$$
\frac{\frac{\Delta I_{S S P}^{(2)}(t=0)}{I_{S S P}^{(2)}(-\infty)}}{\frac{\Delta I_{S P S}^{(2)}(t=0)}{I_{S P S}^{(2)}(-\infty)}}=\frac{\frac{\langle\cos \theta(t=0)\rangle-c_{X X Z}\left\langle\cos ^{3} \theta(t=0)\right\rangle}{\langle\cos \theta(-\infty)\rangle-c_{X X Z}\left\langle\cos ^{3} \theta(-\infty)\right\rangle}}{\frac{\langle\cos \theta(t=0)\rangle-c_{X Z X}\left\langle\cos ^{3} \theta(t=0)\right\rangle}{\langle\cos \theta(-\infty)\rangle-c_{X Z X}\left\langle\cos ^{3} \theta(-\infty)\right\rangle}}=\frac{\frac{\langle\cos \theta(-\infty)\rangle-\left\langle\cos ^{3} \theta(-\infty)\right\rangle-c_{X X Z}\left(\left\langle\cos ^{3} \theta(-\infty)\right\rangle-\left\langle\cos ^{5} \theta(-\infty)\right\rangle\right)}{\langle\cos \theta(-\infty)\rangle-c_{X X Z}\left\langle\cos ^{3} \theta(-\infty)\right\rangle}}{\frac{\langle\cos \theta(-\infty)\rangle-\left\langle\cos ^{3} \theta(-\infty)\right\rangle-c_{X Z X}\left(\left\langle\cos ^{3} \theta(-\infty)\right\rangle-\left\langle\cos ^{5} \theta(-\infty)\right\rangle\right)}{\langle\cos \theta(-\infty)\rangle-c_{X Z X}\left\langle\cos ^{3} \theta(-\infty)\right\rangle}} .
$$

Knowing $D(-\infty)$ and the left hand side term in Eq. (14) enables us to extract $\frac{\left\langle\cos ^{3} \theta(-\infty)\right\rangle}{\left\langle\cos ^{5} \theta(-\infty)\right\rangle}$ as reported in our earlier work. ${ }^{17,42,48}$ If we assume the model that molecules at interfaces wobble in a cone, then a tilt angle and half-angle of the cone can be calculated as documented in previous work. On calculation of the tilt angle and the half-angle, the following terms $\langle\cos \theta(t=0)\rangle,\left\langle\cos ^{3} \theta(t\right.$ $=0)\rangle,\langle\cos \theta(-\infty)\rangle$, and $\left\langle\cos ^{3} \theta(-\infty)\right\rangle$ can be extracted and thereby the pre-factor $\frac{2 \sigma|\mu|^{2}|E|^{2}}{N_{g}(-\infty)}$ in Eq. (13) can be obtained.

Knowing the pre-factor, $\langle\cos \theta(-\infty)\rangle$ and $\left\langle\cos ^{3} \theta(-\infty)\right\rangle$, the two time-correlation functions $\langle\cos \theta(\mathrm{t})\rangle$ and $\left\langle\cos ^{3} \theta(\mathrm{t})\right\rangle$ were obtained. Thus, we are able to write time-dependent order parameter $D(\mathrm{t})$,

$$
D(t)=\frac{\langle\cos \theta(t)\rangle}{\left\langle\cos ^{3} \theta(t)\right\rangle} .
$$

From $\langle\cos \theta(t)\rangle$ and $\left\langle\cos ^{3} \theta(t)\right\rangle$, we obtain the time-dependent average orientational angle $\langle\theta\rangle$ at each time delay. From this, the rate of interfacial orientational motions for molecules at interfaces is obtained.

\section{EXPERIMENTAL SECTION}

The experimental details of the visible pump-vibrational SFG probe measurements have been introduced in previous papers. ${ }^{31,32}$ Briefly, an $800 \mathrm{~nm}$ regeneratively amplified Ti:sapphire system (Spitfire, Spectra Physics) seeded with a MaiTai $80 \mathrm{MHz}, 80 \mathrm{fs}$ oscillator, at $1 \mathrm{kHz}$ repetition frequency was employed in the present experiments. A reflected $20 \%$ output from the regenerative amplifier was used to produce a picosecond $400 \mathrm{~nm}$ pulse. ${ }^{31,32} \mathrm{With}$ difference frequency generation in a $1 \mathrm{~mm} \mathrm{AgGaS} 2$ crystal, tunable infrared lights between $3 \mu \mathrm{m}$ and $8.5 \mu \mathrm{m}$ with a bandwidth of about $150 \mathrm{~cm}^{-1}$ (FWHM) were generated from $45 \%$ of the output power. The remaining $35 \%$ of the $800 \mathrm{~nm}$ femtosecond pulse is introduced into another optical parametric amplifier (OPA) to generate a $1.68 \mu \mathrm{m}$ idler light. The final femtosecond $409 \mathrm{~nm}$ light of $0.5 \mu \mathrm{J}$ was produced by fourth harmonic generation of the idler light from the second OPA as a pump. The pump wavelength was chosen to be close to the absorption maximum of $\mathrm{C} 153$ in solution cite. $^{55-57}$
The IR beam with a typical power of $1.5 \mu \mathrm{J}$ per pulse at $5.7 \mu \mathrm{m}$ and $0.5 \mu \mathrm{J}$ per pulse at $7.7 \mu \mathrm{m}$ was focused onto the sample by a $\mathrm{BaF}_{2}$ lens with a $100 \mathrm{~mm}$ focal length at an angle of $67^{\circ}$ relative to the surface normal with a spot size of around $120 \mu \mathrm{m}$. The $400 \mathrm{~nm}$ picosecond light was focused to $210 \mu \mathrm{m}$ spot size by a $\mathrm{BaF}_{2}$ lens with a $250 \mathrm{~mm}$ focal length at an angle of $76^{\circ}$ from the surface normal. The $409 \mathrm{~nm}$ pump light was focused on the sample from the top by a $\mathrm{BaF}_{2}$ lens with a $500 \mathrm{~mm}$ focal length along the surface normal with a focal spot of $800 \mu \mathrm{m}$. The polarization of the circularly polarized pump beam was controlled using a quarter wave plate.

A $300 \mathrm{~mm}$ spectrograph with one entry and two exits (Acton Research, three gratings including 1200 grooves $/ \mathrm{mm}$ with $450 \mathrm{~nm}$ blazed, 1200 grooves $/ \mathrm{mm}$ with $500 \mathrm{~nm}$ blazed and 600 grooves $/ \mathrm{mm}$ with $4 \mu \mathrm{m}$ blazed) was used. The $450 \mathrm{~nm}$ blazed grating was chosen to measure SFG signals. One exit was configured for detecting the dispersed signal with a liquid-nitrogen cooled, backthinned charged coupled device (CCD) camera (Roper Scientific, $1340 \times 400$ pixels) operating at $-120^{\circ} \mathrm{C}$. The generated SFG signal was focused into the monochromator and detected by a photomultiplier tube (PMT) (Hamamatsu) when the time-profile experiments were performed. The signal from PMT was sent into a BOXCAR averager and then into a lock-in amplifier with reference to a $500 \mathrm{~Hz}$ chopper frequency in the pump light arm. Two probe beams were guaranteed to overlap temporally at the maximum SFG signals in all the experiments. A translational stage and the signal sampling from the lock-in amplifier were controlled by a Labview program.

The purity of C153 was checked to be $99.0 \%$ by highperformance liquid chromatography (HPLC) and used without further purification. The Gibbs monolayers of $\mathrm{C} 153$ were prepared by allowing the $\mathrm{C} 153$ solution to stand for 30 minutes prior to the start of laser experimentation in order to allow for complete formation of the monolayers. The Teflon beakers were cleaned prior to use by immersion in freshly prepared piranha solution for $20 \mathrm{~min}$ and then rinsed with copious amounts of ultrapure water in order to remove any trace organics. Surface tension measurements were performed with a Wilhelmy plate method. The sample was contained in a shallow Teflon beaker mounted on a stage rotating at $2.5 \mathrm{rpm}$ to minimize heating and degradation effects in SFG experiments. The 
SFG signal level from a probe alone was checked to ensure whether samples were degraded over the time. The typical time for a single forward and backward scan was $10 \mathrm{~min}$. After three cycles, it was found that the probe SFG signal had not changed. We found that after five cycles (about $50 \mathrm{~min}$ ), the signal level decreased by $10 \%$. To ensure no photo-degradation of samples, a fresh sample was always made after $30 \mathrm{~min}$; namely, three cycles of forward and backward scans.

\section{RESULTS AND DISCUSSION}

\section{A. Surface excess of $\mathrm{C} 153$ at the air/water interface}

To obtain information on the population of $\mathrm{C} 153$ at the air/water interface, we used Gibb's adsorption equation, which is given $\mathrm{by}^{1,2}$

$$
\Gamma=-\frac{1}{R T} \frac{\partial \gamma}{\partial \ln c}
$$

where $\Gamma$ is the surface excess of $\mathrm{C} 153, c$ is the $\mathrm{C} 153$ concentration in solution, and $\gamma$ is the surface tension. The surface tension as a function of $\mathrm{C} 153$ concentration is shown in Fig. 2. The surface excess at bulk concentration $c$ is obtained by taking a derivative of the surface tension at each concentration, which thereby yields the moles of C153 per unit area. The concentration of a typical SFG experiment was $8 \mu \mathrm{m}$, which corresponds to a surface area of around $660 \AA^{2}$.

\section{B. SFG measurements of $\mathrm{C} 153$ at the air/water interface}

Figure 3(a) shows the SFG spectra of an $8 \mu \mathrm{m} \mathrm{C153}$ aqueous solution taken at the four different polarization combinations SSP,

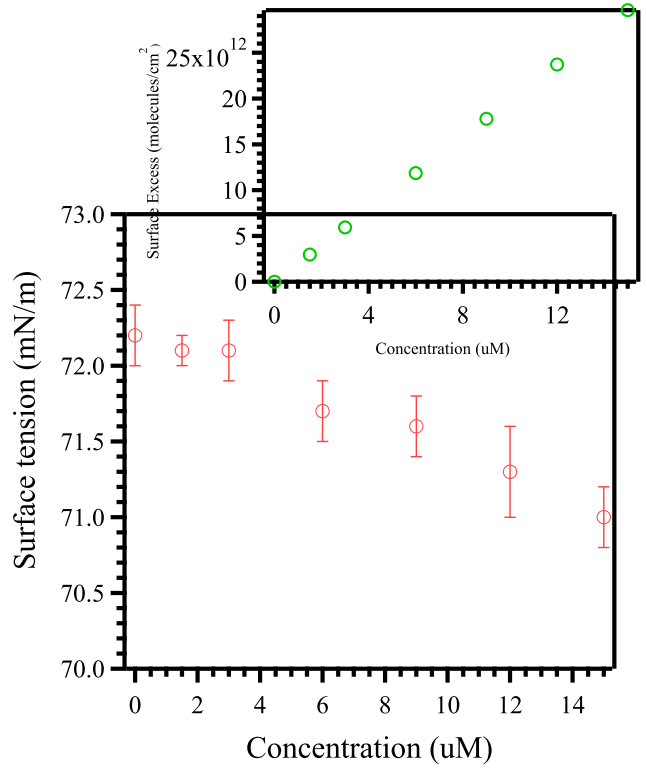

FIG. 2. Surface tension vs. C153 concentration in aqueous solution. The surface excess calculated is shown in the inset.
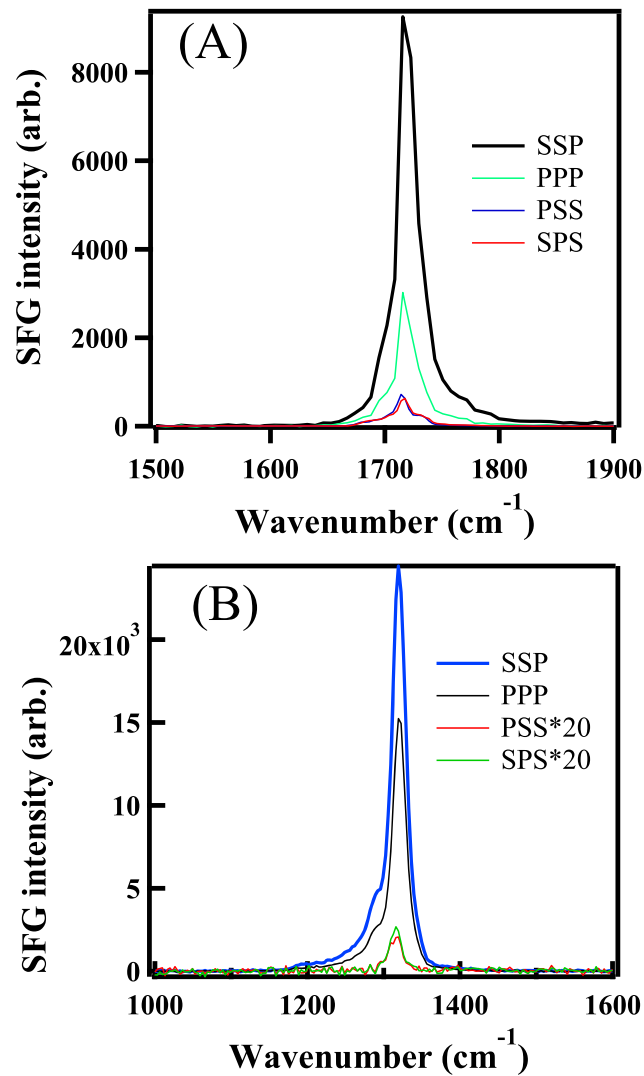

FIG. 3. (a) The SFG spectra of $8 \mu \mathrm{m} \mathrm{C} 153$ for four different polarization combinations (SSP, PPP, SPS, and PSS). In (a), the peak at $1718 \mathrm{~cm}^{-1}$ is assigned to the stretching mode of the $-\mathrm{C}=0$ group of $\mathrm{C} 153$. In (b), the peak at $1319 \mathrm{~cm}^{-1}$ is assigned to the stretching mode of the $-\mathrm{CF}_{3}$ group of $\mathrm{C} 153$.

$P P P, S P S$, and PSS with the visible beam at $400 \mathrm{~nm}$ and the IR centered at $1700 \mathrm{~cm}^{-1}$ with a bandwidth of $150 \mathrm{~cm}^{-1}$. There is only one peak observed at $1718 \mathrm{~cm}^{-1}$ in all the polarization spectra. This peak was assigned to the stretching mode of the $-\mathrm{C}=\mathrm{O}$ group of $\mathrm{C} 153$. It was found that the SFG intensities differ for the different polarization combinations. The SFG signals of the SSP polarization are almost three times stronger than those of the $P P P$ polarization. The intensities of the SPS and PSS polarization are almost identical, being nearly ten times weaker than those of the SSP polarization. These ratios are used to find the orientation angle of the $-\mathrm{C}=\mathrm{O}$ group of C153.

Figure 3(b) shows the SFG spectra of $8 \mu \mathrm{m} \mathrm{C153}$ aqueous solution taken at the four different polarization combinations SSP, PPP, SPS, and PSS with the visible beam at $400 \mathrm{~nm}$ and the IR centered at $1300 \mathrm{~cm}^{-1}$ with a bandwidth of $150 \mathrm{~cm}^{-1}$. The main peak at $1319 \mathrm{~cm}^{-1}$ appears in the four polarization spectra. We assigned the peak to the symmetric stretching mode of $-\mathrm{CF}_{3}$ in the ring of C153. ${ }^{58}$ The SFG intensity for the SSP polarization is slightly stronger than that of the PPP polarization. The SFG intensities for the SPS and PSS polarization are almost identical, being nearly 100 times weaker than those of the SSP polarization. These ratios are used to find the orientation angle of the $-\mathrm{CF}_{3}$ group of $\mathrm{C} 153$. 


\section{Absolute orientational structure of $\mathrm{C} 153$ at the air/water interface}

The approach that we employed to determine absolute orientational structure was established in earlier work. ${ }^{42}$ Briefly measurements of the intensity ratio between the different polarization combinations yielded the interfacial orientational order parameter $D(-\infty)$ prior to the pump pulse, as shown in Eq. (7). From the order parameter $D(-\infty)$, the average orientational angles of the $-\mathrm{C}=\mathrm{O}$ group and $-\mathrm{CF}_{3}$ groups of $\mathrm{C} 153$ with respect to the surface normal were extracted. Based on the relative orientation of the two groups in the C153 molecule and the SFG measured orientations of the two groups, the absolute orientation of the C153 molecule can be found.

Using the intensity ratios between the different polarization combinations shown in Figs. 3(a) and 3(b) and using Eq. (7), we found the orientational angles of the $-\mathrm{C}=\mathrm{O}$ and $-\mathrm{CF}_{3}$ groups to be $144^{\circ}$ and $59^{\circ}$ with respect to the surface normal assuming a narrow orientational distribution. Combining these two measurements with the known angle of $120^{\circ}$ between the $-\mathrm{C}=\mathrm{O}$ and $-\mathrm{CF}_{3}$ symmetric stretch axes, we obtained the absolute orientation of the $\mathrm{C} 153$ molecules at the air/water interface. The angle of the normal to the molecular plane with respect to the interface normal was found to be $55^{\circ}$, which is also the angle between the molecular plane and the surface plane. In Fig. 4, the orientational structure of C153 at the air/water interface is shown.

\section{Orientational dynamics of the individual $-\mathrm{C}=\mathrm{O}$ and $-\mathrm{CF}_{3}$ groups}

Figure 5(a) shows the time traces for SFG intensities of the $-\mathrm{C}=\mathrm{O}$ group in the $\mathrm{C} 153$ molecule for both the SSP and SPS polarization combinations, respectively. This figure plots the ratio of the normalized SFG intensities to the SFG intensities at $t<0$ $(\mathrm{I}(\mathrm{t}) / \mathrm{I}(-\infty))$ versus the time delay of the pump pulse (with respect to the probe pulses). The solid lines of these traces were fitted to a single exponential that gives a recovery time of $105 \pm 11$ ps for the SSP combination and a recovery time of $62 \pm 5$ ps for the SPS combination. These recovery times differ for the two polarization combinations because of the different sensitivity to the

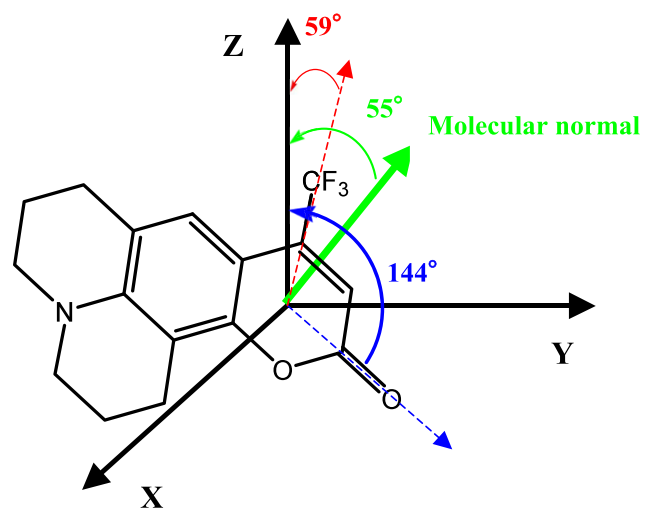

FIG. 4. An absolute orientational configuration of $\mathrm{C} 153$ at the air/water interface. The orientational angles for the $-\mathrm{C}=\mathrm{O},-\mathrm{CF}_{3}$, and the molecular normal are $144^{\circ}, 59^{\circ}$, and $55^{\circ}$, respectively.
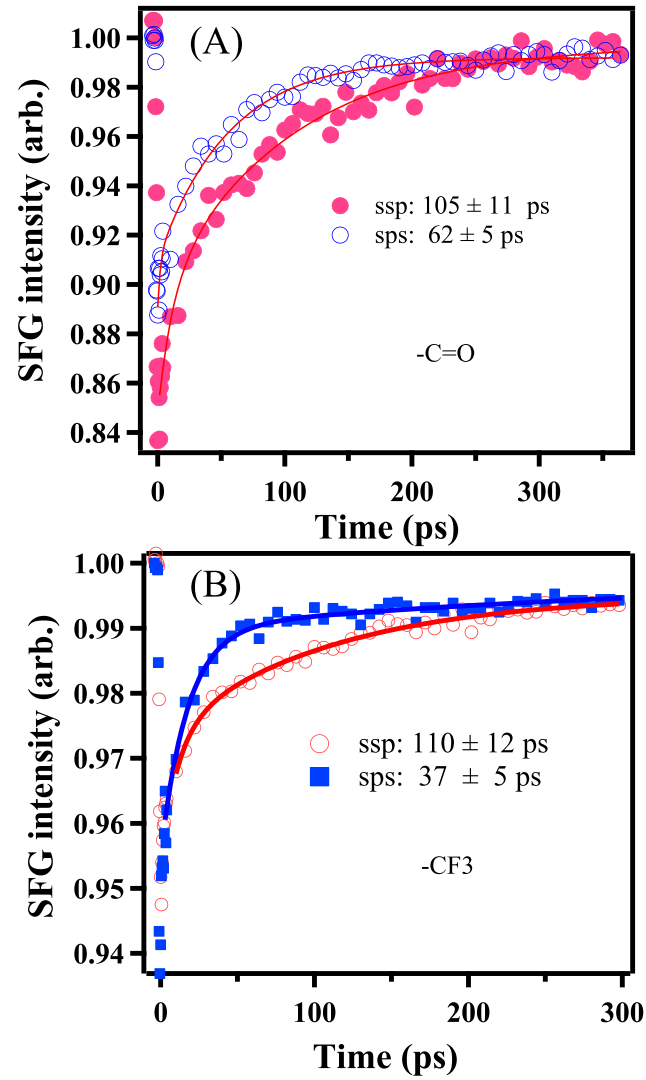

FIG. 5. The time traces of SFG intensities of SSP and SPS for the $-\mathrm{C}=0$ group (a) and the $-\mathrm{CF}_{3}$ group (b) with respect to the SFG intensities at $t<0$ after photo-excitation of a circularly polarized pump incident along the surface normal.

pump-induced non-equilibrium orientational distribution for each polarization combination. The time-dependent responses for different polarization combinations are a result of different weighted sums of $\langle\cos \theta(t)\rangle$ and $\left\langle\cos ^{3} \theta(t)\right\rangle$, as shown in Eq. (13). These recovery times were attributed to out-of-plane orientational relaxation time of the $-\mathrm{C}=\mathrm{O}$ group of $\mathrm{C} 153$ at the air/water interface.

Figure 5(b) displays the time traces for the SFG intensities of the $-\mathrm{CF}_{3}$ group in the C153 molecule for both the SSP and SPS polarization combinations. Using the methods employed for Fig. 5(a), we found that the recovery time of $S S P$ is $110 \pm 12$ ps and the recovery time of the SPS is considerably faster being $37 \pm 5$ ps.

\section{E. Absolute orientational dynamics}

Figures 6(a) and 6(b) show the time-dependence of the orientational angles of the $-\mathrm{C}=\mathrm{O}$ and $-\mathrm{CF}_{3}$ groups in the $\mathrm{C} 153$ molecule. Upon excitation at $\mathrm{t}=0$, the vibrational $-\mathrm{C}=\mathrm{O}$ dipole moment rotates from $148^{\circ}$ back to the measured ground state equilibrium orientational angle of $144^{\circ}$ (the $-\mathrm{C}=\mathrm{O}$ angle before photoexcitation). The time constant for this orientational relaxation was found to be $130 \pm 20 \mathrm{ps}$. The $-\mathrm{CF}_{3}$ group exhibited a rotation from $65^{\circ}$ back to our measured ground state equilibrium orientation angle of $59^{\circ}$ with a time constant of $210 \pm 38$ ps. Once these time-dependent 


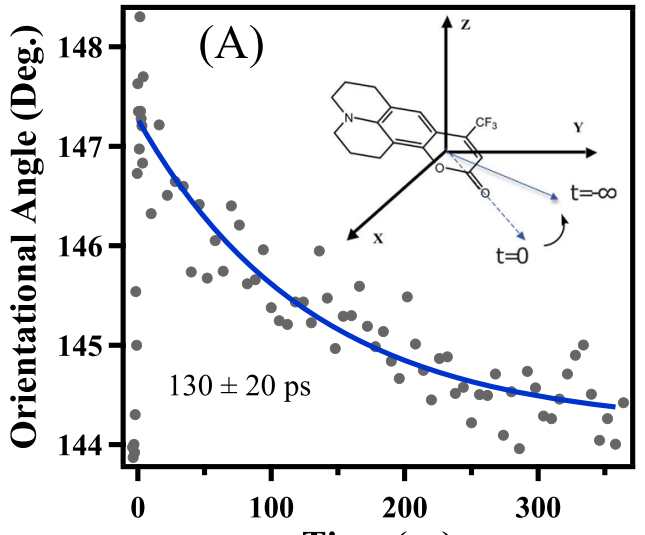

Time (ps)

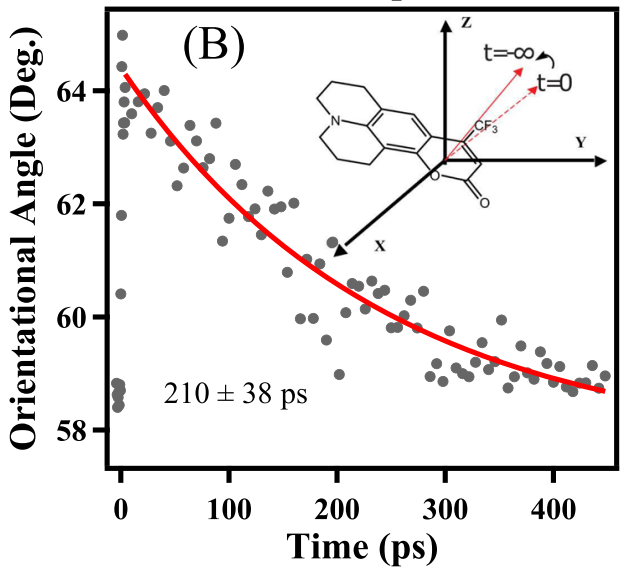

FIG. 6. The time-dependent average orientational angle for the $-\mathrm{C}=0$ group shown in (a) and the $-\mathrm{CF}_{3}$ group shown in (b).

orientational angles of $-\mathrm{C}=\mathrm{O}$ are known, the orientational motions of the normal to the molecular plane of $\mathrm{C} 153$ with respect to the surface normal were found. The angle of the normal to the molecular plane with respect to the interface normal as a function of time is shown in Fig. 7. When excited, the normal to the molecular plane rotates from its pump induced non-equilibrium value of $58^{\circ}$ to its ground state equilibrium value of $55^{\circ}$, with a time constant of $125 \pm 26$ ps.

\section{F. Interfacial orientational diffusion}

For orientational relaxation in bulk liquids, the evolution function can be obtained from an orientational diffusion model. Due to the complexity of rotational motions in the anisotropic environment of an interface, we do not, at this time, have a model for molecular motions in an interfacial region. However, the wobbling-in-a-cone model has had some success in describing the orientational dynamics in restricted environments. ${ }^{41,42,59-64}$ The basic idea for the wobbling-in-a-cone model is that the motion of the probe molecule is described as diffusive ("wobbling") within a cone of a certain semiangle and diffusion outside the cone is forbidden. Within the framework of this model, an analytical expression of the diffusion coefficient for wobbling that relates the relaxation time to the semi-angle

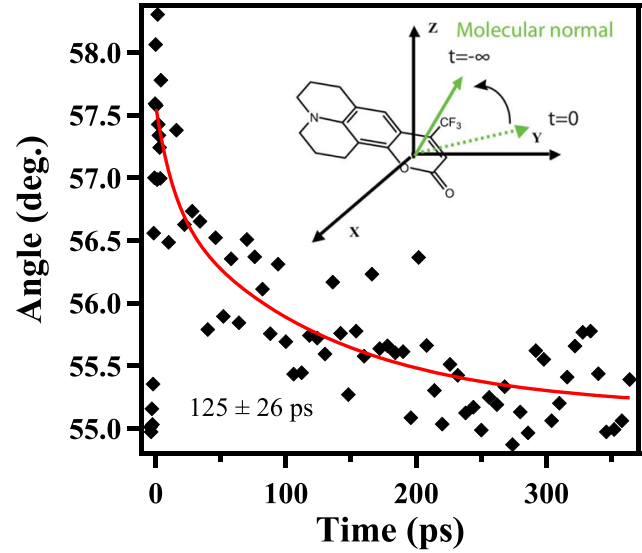

FIG. 7. The time-dependent average orientational angle for the molecular normal to the molecular plane of $\mathrm{C} 153$.

of the cone. Although this model is simple, it has been successfully applied to rotational diffusion of lipids and proteins in a lipid matrix. Based on the model, the interfacial orientational diffusion coefficient $D_{\theta}$ is expressed as $D_{\theta}=\frac{1}{2} \lim _{t \rightarrow \infty} \frac{\left\langle\left[\theta_{t}-\theta_{t=0}\right]\right\rangle^{2}}{t}=\frac{1}{2} \frac{\left\langle\Delta \theta^{2}\right\rangle}{\tau}$, where $\Delta \theta$ is the maximum change in the orientational angle upon photo-excitation and $\tau$ is a measured orientational relaxation time. On application of this model, we found that the interfacial orientational diffusion coefficients for the $-\mathrm{C}=\mathrm{O}$ and $-\mathrm{CF}_{3}$ groups are $9.4 \times 10^{-6} \mathrm{ps}^{-1}$ and $2.2 \times 10^{-5} \mathrm{ps}^{-1}$, which are two orders of magnitude slower than rotational diffusion of $\mathrm{C} 153$ in bulk water. ${ }^{59,65}$ It is important to note that the changes in the orientational angles for $-\mathrm{C}=\mathrm{O}$ and $-\mathrm{CF}_{3}$ have been obtained for a delta orientational distribution. The orientational distribution for $-\mathrm{C}=\mathrm{O}$ and $-\mathrm{CF}_{3}$ groups was found to be $4.5^{\circ}$ and $6.8^{\circ}$, respectively. Previous SHG studies also showed that Coumarin $314(\mathrm{C} 314)$ at the air/water interface has a narrow orientational distribution width of $16^{\circ}$, which corresponds to a half angle of $8^{\circ}$ for the case of wobbling diffusion. These results indicate that $\mathrm{C} 153$ and C314 molecules are well ordered at the interface through interactions with interfacial water solvents. Although the cone model used above is an oversimplification of the real potential energy of interfacial molecules in the interfacial environment, our findings suggest that at the air/water interface $\mathrm{C} 153$ molecules experience a much stronger out-of-plane friction than the friction in bulk water.

A molecular dynamics calculation found that orientational motions in the interfacial region depend strongly on the polarity and location of the adsorbates. ${ }^{66}$ Molecular dynamics simulations of C314 at the air/water interface yielded a number of equilibrium and dynamics properties. ${ }^{67}$ The calculated orientation of C314 is in good agreement with experimental results. Calculations of the ground and excited state dipole moments were also found to be in reasonable agreement with the measured values. However, simulations of dynamic processes for $\mathrm{C} 314$ at the air/aqueous interface are more difficult to carry out. The out-of-plane rotational correlation relaxation times obtained from simulations were roughly an order of magnitude faster than the experimental results found in the work reported here. This discrepancy can be attributed to the simulation correlation time expression that involves the $\langle\cos \theta(t) \cos \theta(0)\rangle$ 
time correlation function, whereas the SHG and SFG time correlation functions involve terms such as $\left\langle\cos ^{3} \theta(t) \cos ^{2} \theta(0)\right\rangle$ as well as $\left\langle\cos \theta(t) \cos ^{2} \theta(0)\right\rangle .^{67}$ It would be of value to carry out a simulation for the rotational correlation function using terms such as $\left\langle\cos ^{3} \theta(t) \cos ^{2} \theta(0)\right\rangle$ and $\left\langle\cos \theta(t) \cos ^{2} \theta(0)\right\rangle$, which describe the temporal correlation function obtained from SHG and SFG measurements. Even though a new simulation would be useful, the orientation calculated from our simplified model still can give the orientation of $\mathrm{C} 153$ at the interface in three dimensions. The technique we used can help study interfacial interactions as well as study molecular motions during chemical reactions that occur at an interface.

\section{CONCLUSIONS}

We have measured the absolute 3 dimensional rotational motions of Coumarin $153(\mathrm{C} 153)$ at an air/water interface using the time-resolved polarized Sum Frequency Generation (TR-SFG) technique. To begin with the equilibrium orientations of the symmetry axes of the $-\mathrm{C}=\mathrm{O}$, the $\mathrm{CF}_{3}$ groups with respect to the interface normal were measured when $\mathrm{C} 153$ was in its ground electronic state. By perturbing the equilibrium orientational distribution of $\mathrm{C} 153$, we then measured the time dependence of the absolute orientations of the two chromophores as the $\mathrm{C} 153$ molecules rotate toward their equilibrium orientations. Perturbation of the equilibrium orientational distribution was achieved by photoexciting a subset of the equilibrium distribution with a polarized pump pulse that is resonant with the lowest excited singlet state of $\mathrm{C} 153$. The polarization of the pump pulse determines the contribution of in-plane and out-of-plane rotations to the measured SFG intensity. Using a circularly polarized pump pulse that was incident normal to the interface enabled us to separate the out of-plane from the in-plane rotations. The photoselection process using the circularly polarized pump pulse shifted the average orientation of the $\mathrm{C}=\mathrm{O}$ group by an increase of $4^{\circ} \pm 0.6^{\circ}$. The rotational recovery time was found to be $130 \pm 20 \mathrm{ps}$, which is the time to reach the equilibrium orientational distribution. The orientational change in the normal to the molecular plane was found to be $4^{\circ} \pm 0.5^{\circ}$ with a rotational recovery time of $125 \pm 26$ ps. It is also noted that, as given above, the transient shift in orientation and the orientational relaxation dynamics of the $-\mathrm{C}=\mathrm{O}$ group and the angle of the normal to the molecular plane with respect to the normal of the interfacial plane are the same. We believe that these observations act as guidance in understanding bond-making and bond-breaking in photochemistry, in particular, spatial selectivity of chemical reactions at interfaces and surfaces.

\section{SUPPLEMENTARY MATERIAL}

See supplementary material for time-dependent SFG responses under SSP and PPP for the $-\mathrm{C}=\mathrm{O}$ group and the $-\mathrm{CF}_{3}$ group of coumarin 153 at the air/water interface.

\section{ACKNOWLEDGMENTS}

K.B.E. is thankful for generous support of this research through Award No. CHE-1360969. Y.R. is thankful for generous support of this research through Award No. CHE-1506789. The authors also thank Dr. Michael Fayer, Dr. Tony F. Heinz, and Dr. Dezheng Sun for their helpful discussions.

The authors declare no competing financial interest

\section{REFERENCES}

${ }^{1}$ P. B. Miranda and Y. R. Shen, J. Phys. Chem. B 103, 3292 (1999).

${ }^{2}$ Y. R. Shen, Annu. Rev. Phys. Chem. 40, 327 (1989).

${ }^{3}$ Y. R. Shen, The Principles of Nonlinear Optics (John Wiley \& Sons, Inc., Hoboken, New Jersey, 2003).

${ }^{4}$ K. B. Eisenthal, Chem. Rev. 96, 1343 (1996).

${ }^{\mathbf{5}}$ F. M. Geiger, Annu. Rev. Phys. Chem. 60, 61 (2009).

${ }^{6}$ A. L. Mifflin, M. J. Musorrafiti, C. T. Konek, and F. M. Geiger, J. Phys. Chem. B 109, 24386 (2005).

${ }^{7}$ O. Esenturk and R. A. Walker, J. Chem. Phys. 125, 174701 (2006).

${ }^{8}$ J. T. Fourkas, R. A. Walker, S. Z. Can, and E. Gershgoren, J. Phys. Chem. C 111, 8902 (2007).

${ }^{9}$ Y. B. Fan, X. Chen, L. J. Yang, P. S. Cremer, and Y. Q. Gao, J. Phys. Chem. B 113, 11672 (2009).

${ }^{10}$ S. Kataoka and P. S. Cremer, J. Am. Chem. Soc. 128, 5516 (2006).

${ }^{11}$ X. Y. Chen, H. Z. Tang, M. A. Even, J. Wang, G. N. Tew, and Z. Chen, J. Am. Chem. Soc. 128, 2711 (2006).

${ }^{12}$ J. C. Conboy and M. A. Kriech, Anal. Chim. Acta 496, 143 (2003).

${ }^{13}$ M. J. Shultz, S. Baldelli, C. Schnitzer, and D. Simonelli, J. Phys. Chem. B 106, 5313 (2002).

${ }^{14}$ S. Baldelli, Acc. Chem. Res. 41, 421 (2008).

${ }^{15}$ M. Xu, R. Spinney, and H. C. Allen, J. Phys. Chem. B 113, 4102 (2009).

${ }^{16}$ W. Gan, D. Wu, Z. Zhang, R. R. Feng, and H. F. Wang, J. Chem. Phys. 124, 114705 (2006).

${ }^{17}$ H. F. Wang, W. Gan, R. Lu, Y. Rao, and B. H. Wu, Int. Rev. Phys. Chem. 24, 191 (2005).

${ }^{18}$ J. A. Carter, Z. H. Wang, and D. D. Dlott, Acc. Chem. Res. 42, 1343 (2009).

${ }^{19}$ Z. H. Wang, D. G. Cahill, J. A. Carter, Y. K. Koh, A. Lagutchev, N. H. Seong, and D. D. Dlott, Chem. Phys. 350, 31 (2008).

${ }^{20}$ M. Sovago, E. Vartiainen, and M. Bonn, J. Chem. Phys. 131, 161107 (2009).

${ }^{21}$ G. J. Simpson, J. M. Perry, and C. L. Ashmore-Good, Phys. Rev. B 66, 165437 (2002).

${ }^{22}$ L. Fu, J. Liu, and E. C. Y. Yan, J. Am. Chem. Soc. 133, 8094 (2011).

${ }^{23}$ A. D. Curtis, M. C. Asplund, and J. E. Patterson, J. Phys. Chem. C 115, 19303 (2011).

${ }^{24}$ J. E. Laaser, W. Xiong, and M. T. Zanni, J. Phys. Chem. B 115, 2536 (2011).

${ }^{25}$ A. V. Benderskii, J. Henzie, S. Basu, X. M. Shang, and K. B. Eisenthal, J. Phys. Chem. B 108, 14017 (2004).

${ }^{26}$ K. T. Nguyen, X. M. Shang, and K. B. Eisenthal, J. Phys. Chem. B 110, 19788 (2006).

${ }^{27}$ Y. Rao, S. Y. Hong, N. J. Turro, and K. B. Eisenthal, J. Phys. Chem. C 115, 11678 (2011).

${ }^{28}$ X. M. Shang, K. Nguyen, Y. Rao, and K. B. Eisenthal, J. Phys. Chem. C 112, 20375 (2008).

${ }^{29}$ X. Shi, E. Borguet, A. N. Tarnovsky, and K. B. Eisenthal, Chem. Phys. 205, 167 (1996).

${ }^{30}$ D. Zimdars, J. I. Dadap, K. B. Eisenthal, and T. F. Heinz, J. Phys. Chem. B 103, 3425 (1999).

${ }^{31}$ Y. Rao, N. J. Turro, and K. B. Eisenthal, J. Phys. Chem. C 114, 17703 (2010).

${ }^{32}$ Y. Rao, D. H. Song, N. J. Turro, and K. B. Eisenthal, J. Phys. Chem. B 112, 13572 (2008).

${ }^{33}$ J. A. McGuire and Y. R. Shen, Science 313, 1945 (2006).

${ }^{34}$ P. Sen, S. Yamaguchi, and T. Tahara, Faraday Discuss. 145, 411 (2010).

${ }^{35}$ C.-S. Hsieh, R. K. Campen, A. C. Vila Verde, P. Bolhuis, H.-K. Nienhuys, and M. Bonn, Phys. Rev. Lett. 107, 116102 (2011).

${ }^{36}$ Z. Zhang, L. Piatkowski, H. J. Bakker, and M. Bonn, Nat. Chem 3, 888 (2011).

${ }^{37}$ A. Eftekhari-Bafrooei and E. Borguet, J. Am. Chem. Soc. 132, 3756 (2010).

${ }^{38}$ K. B. Eisenthal, Acc. Chem. Res. 26, 636 (1993). 
${ }^{39} \mathrm{~T}$. Araiso and T. Koyama, Chem. Phys. Lipids 50, 105 (1989).

${ }^{40}$ A. Castro, E. V. Sitzmann, D. Zhang, and K. B. Eisenthal, J. Phys. Chem. 95, 6752 (1991).

${ }^{41}$ M. P. Heitz and F. V. Bright, Appl. Spectrosc. 49, 20 (1995).

${ }^{42}$ Y. Rao, M. Comstock, and K. B. Eisenthal, J. Phys. Chem. B 110, 1727 (2006).

${ }^{43}$ X. Wei, S. C. Hong, X. W. Zhuang, T. Goto, and Y. R. Shen, Phys. Rev. E 62, $5160(2000)$

${ }^{44}$ C. Hirose, N. Akamatsu, and K. Domen, Appl. Spectrosc. 46, 1051 (1992).

${ }^{45}$ C. Hirose, N. Akamatsu, and K. Domen, J. Chem. Phys. 96, 997 (1992).

${ }^{46}$ D. Zhang, J. Gutow, and K. B. Eisenthal, J. Phys. Chem. 98, 13729 (1994).

${ }^{47}$ X. Zhuang, P. B. Miranda, D. Kim, and Y. R. Shen, Phys. Rev. B 59, 12632 (1999).

${ }^{48}$ Y. Rao, Y. S. Tao, and H. F. Wang, J. Chem. Phys. 119, 5226 (2003).

${ }^{49}$ D. Zimdars and K. B. Eisenthal, J. Phys. Chem. B 105, 3993 (2001).

${ }^{50}$ A. V. Benderskii and K. B. Eisenthal, J. Phys. Chem. B 104, 11723 (2000).

${ }^{51}$ R. Lu, W. Gan, B.-h. Wu, H. Chen, and H.-f. Wang, J. Phys. Chem. C 108, 7297 (2004).

${ }^{52}$ R. Lu, W. Gan, B.-h. Wu, Z. Zhang, Y. Guo, and H.-F. Wang, J. Phys. Chem. B 109, 14118 (2004).

${ }^{53}$ Y. Rao, M. Xu, S. Jockusch, N. J. Turro, and K. B. Eisenthal, Chem. Phys. Lett. 544, 1 (2012).
${ }^{54}$ M. B. Raschke, M. Hayashi, S. H. Lin, and Y. R. Shen, Chem. Phys. Lett. 359, 367 (2002).

${ }^{55}$ Y. Jiang, P. K. McCarthy, and G. J. Blanchard, Chem. Phys. 183, 249 (1994).

${ }^{56}$ R. Improta, V. Barone, and F. Santoro, Angew. Chem., Int. Ed. 46, 405 (2007).

${ }^{57}$ S. A. Kovalenko, J. Ruthmann, and N. P. Ernsting, Chem. Phys. Lett. 271, 40 (1997).

${ }^{58}$ C. B. Kristalyn, S. Watt, S. A. Spanninga, R. A. Barnard, K. Nguyen, and Z. Chen, J. Colloid Interface Sci. 353, 322 (2011).

${ }^{59}$ Z. Gengeliczki, D. E. Rosenfeld, and M. D. Fayer, J. Chem. Phys. 132, 244703 (2010).

${ }^{60}$ P. B. Moore, C. F. Lopez, and M. L. Klein, Biophys. J. 81, 2484 (2001).

${ }^{61}$ J. P. Starck, Y. Nakatani, G. Ourisson, D. J. Cowley, and G. Duportail, New J. Chem. 20, 1293 (1996)

${ }^{62}$ M. Tarek, D. J. Tobias, and M. L. Klein, J. Phys. Chem. 99, 1393 (1995).

${ }^{63}$ H. Saito, T. Araiso, H. Shirahama, and T. Koyama, J. Biochem. 109, 559 (1991).

${ }^{64} \mathrm{~N}$. Kimura, R. Tsuneta, T. Araiso, T. Koyama, N. Hasegawa, T. Ishii, and K. Mukasa, Chem. Phys. Lipids 57, 39 (1991).

${ }^{65}$ C. C. Wang and R. Pecora, J. Chem. Phys. 72, 5333 (1980).

${ }^{66}$ M. L. Johnson, C. Rodriguez, and I. Benjamin, J. Phys. Chem. A 113, 2086 (2009).

${ }^{67}$ D. A. Pantano and D. Laria, J. Phys. Chem. B 107, 2971 (2003). 